Published in final edited form as:

Am J Ophthalmol. 2017 April ; 176: 194-202. doi:10.1016/j.ajo.2017.01.010.

\title{
INTRAVITREAL BEVACIZUMAB FOR THE TREATMENT OF VITREOUS HEMORRHAGE DUE TO PROLIFERATIVE DIABETIC RETINOPATHY
}

\author{
Rupin N. Parikh ${ }^{1,2}$, Anastasia Traband ${ }^{1}$, Anton M. Kolomeyer ${ }^{1}$, Brian L. VanderBeek ${ }^{1,3,4}$, \\ Benjamin J. Kim ${ }^{1}$, Albert M. Maguire ${ }^{1}$, and Alexander J. Brucker ${ }^{1}$ \\ ${ }^{1}$ Scheie Eye Institute, Department of Ophthalmology, University of Pennsylvania, 51 North 39th \\ Street, Philadelphia, PA 19104 \\ ${ }^{2}$ Albany Medical College, 47 New Scotland Avenue, Albany, NY 12208 \\ ${ }^{3}$ Center for Clinical Epidemiology and Biostatistics, University of Pennsylvania Perelman School \\ of Medicine, 423 Guardian Drive, 8th Floor Blockley Hall, Philadelphia, Pennsylvania 19104 \\ ${ }^{4}$ Leonard Davis Institute, University of Pennsylvania Perelman School of Medicine, 3641 Locust \\ Walk, Philadelphia, Pennsylvania 19104
}

\section{Abstract}

PURPOSE-To evaluate the outcomes of intravitreal bevacizumab (IVB) use in patients with a vitreous hemorrhage (VH) secondary to proliferative diabetic retinopathy (PDR).

DESIGN-Retrospective, interventional case series.

METHODS-Patients who presented to Scheie Eye Institute between January 2008 and January 2015 with a new VH secondary to PDR and treated with IVB were included. Exclusion criteria consisted of IVB treatment prior to the study, a history of pars plana vitrectomy (PPV), and less than one year of follow-up. Outcomes of interest were additional treatments including PPV, injections, and panretinal photocoagulation (PRP), as well as visual acuity at baseline and one year.

RESULTS-Of the 111 eligible eyes, 55 (49.5\%) had PRP, 35 (31.6\%) were managed with injections alone, and $21(18.9 \%)$ had PPV after one year. The overall average number of injections

Corresponding Author: Alexander J. Brucker, MD, Scheie Eye Institute, University of Pennsylvania, 51 N 39th Street, 5th Floor, Philadelphia, PA 19104, Phone: 1-215-662-9702, Fax: 1-215-243-4696, ajbrucke@ mail.med.upenn.edu.

Conflict of Interest: No conflicting relationship exists for any author.

Portions of this data were presented as a poster presentation the 2016 Association for Research in Vision and Ophthalmology Meeting Disclaimer from corresponding author: I had full access to all the data in the study and take responsibility for the integrity of the data and the accuracy of the data analysis as well as the decision to submit for publication.

\section{DISCLOSURES}

Financial Disclosures: No financial disclosures.

Other Acknowledgments: No other acknowledgments.

Publisher's Disclaimer: This is a PDF file of an unedited manuscript that has been accepted for publication. As a service to our customers we are providing this early version of the manuscript. The manuscript will undergo copyediting, typesetting, and review of the resulting proof before it is published in its final citable form. Please note that during the production process errors may be discovered which could affect the content, and all legal disclaimers that apply to the journal pertain. 
during this time was 2 (range 1-9), and $13(11.7 \%)$ eyes were managed with a single injection alone. Of the 69 eyes with two years of follow up, 43 (62.3\%) had PRP, 16 (23.2\%) were treated with injections alone, and $10(14.5 \%)$ had PPV.

CONCLUSIONS-This study underscores the potentially important role that IVB injections have in the management of patients with VH secondary to PDR. The results indicate that a proportion of patients may be treated with a minimal amount of intervention requiring one or two antivascular endothelial growth factor injections only. Also, the rate of PPV at two years (27.9\%, $\mathrm{n}=31$ ) suggests that most patients may be managed non-surgically.

\section{INTRODUCTION}

Diabetic retinopathy is the leading cause of severe vision loss in working aged adults worldwide ${ }^{1}$. Proliferative diabetic retinopathy (PDR) is a progressive condition due to retinal ischemia and is characterized by the development of retinal neovascularization. These abnormal and incompetent blood vessels can grow along the surface of the vitreous and may lead to bleeding into the vitreous cavity or fibrovascular proliferation that can result in tractional retinal detachments (TRD) ${ }^{2}$.

For years, panretinal photocoagulation (PRP) has been the standard of care for high risk PDR and has been shown to reduce severe vision loss by more than $50 \%{ }^{1}$. Unfortunately, PRP is not without its drawbacks. It is inherently destructive to the peripheral retina and can lead to adverse effects on visual function including loss of peripheral vision and nyctalopia ${ }^{3}$. Additionally, a small percentage of patients may continue to progress despite a full laser treatment. If vitreous hemorrhage $(\mathrm{VH})$ occurs due to PDR prior to detection of the neovascularization, it may prevent PRP treatment by obscuring the retina. In these cases, if the VH does not clear on its own, pars plana vitrectomy (PPV) becomes necessary exposing the patient to a new set of potential complications ${ }^{4}$. Furthermore, surgery alone does not preclude the possibility of recurrent $\mathrm{VH}$, which has been reported to occur in $20-40 \%$ of eyes following vitrectomy for non-clearing $\mathrm{VH}(\mathrm{NCVH})^{5}$.

Hypoxic retinal tissue induces the increased production of the angiogenic agent vascular endothelial growth factor (VEGF), which plays a major role in the development of neovascularization ${ }^{3}$. Bevacizumab is a humanized monoclonal antibody that is active against all VEGF-A isoforms ${ }^{3}$. Anti-VEGF therapy has been shown to cause regression of neovascularization and to potentially prevent further hemorrhaging ${ }^{6,11,13}$. Combination bevacizumab and PRP therapy for PDR has already been found to be effective in promoting regression of neovascularization ${ }^{1,3}$.

To date, minimal literature exists on the effect of using intravitreal anti-VEGF injections alone as first-line treatment for VH in patients with PDR. A recent study from the Diabetic Retinopathy Clinical Research (DRCR) Network presented results from a prospective trial comparing outcomes in patients with PDR that were either treated with ranibizumab (another anti-VEGF compound) or PRP ${ }^{10}$. This study found that VA outcomes with ranibizumab treatment were non-inferior to PRP treatment at the end of two years ${ }^{10}$. While this study revealed a potential role for anti-VEGF treatment in patients with PDR, it did not specifically address its role in patients presenting with $\mathrm{VH}$. It is also unclear whether, in the 
absence of PRP treatment, if periodic intravitreal injections will be necessary indefinitely to suppress neovascularization. An earlier DRCR study compared the outcome of intravitreal ranibizumab (IVR) injections versus intravitreal saline injections in the treatment of VH in patients with PDR, and suggested that IVR increased the likelihood of completion of PRP without PPV ${ }^{7,8}$. However, due to the study's relatively short follow up of 16 weeks, the duration of treatment effect was indeterminate, and the authors concluded that a clinically significant difference in outcomes between IVR and intravitreal saline could not be determined ${ }^{9}$.

Several studies have explored the role of anti-VEGF in PDR $^{13}$, but the long-term efficacy, potential need for frequent injections, and sustainability of neovascular regression remain unclear. In addition, clinical trial regimens are not always possible in real world situations due to the potential care burden they can impose, at times necessitating alternative approaches. Confirming that real world treatment regimen modifications are not inferior to the clinical trial standard then becomes critical. This study attempts to better understand the natural history and response of VH after anti-VEGF treatment in patients with PDR. Of particular interest are any additional treatments performed over the study period including PPV, PRP, or additional injections.

\section{METHODS}

\section{Study Design}

This study adhered to the tenets of the Declaration of Helsinki and all of the United States federal and Pennsylvania state laws. It was conducted with the approval of the University of Pennsylvania's Institutional Review Board for chart review. We performed a retrospective interventional case series of patients who presented to the Scheie Eye Institute between July 2010 and November 2015. A list of potential study subjects was compiled by searching the University of Pennsylvania's electronic patient database using the keywords "vitreous hemorrhage," "proliferative diabetic retinopathy," "avastin," and "bevacizumab." Once potential study subjects were identified, a chart review was performed to collect any treatments (intravitreal injection, laser, or surgery) through one year of follow-up. Two-year follow-up data were also collected when available for eyes that did not require PPV during the first year.

\section{Study Population}

The study included subjects over the age of 18 with a history or diagnosis of PDR in at least one eye and VH who received intravitreous bevacizumab (IVB). All eligible eyes were examined by at least one of the four retinal specialists at the Scheie Eye Institute. Eyes were excluded for any history of IVB treatment, PPV for any indication prior to presentation with a new $\mathrm{VH}$, or evidence of a pre-existing TRD on exam or ultrasound. Subjects who had VH for reasons other than PDR (e.g., sickle cell, retinal vein occlusion, hemorrhagic posterior vitreous detachment) were also excluded. Additional exclusion criteria included neovascular glaucoma (NVG) or diabetic macular edema (DME). Since this is a natural history study of IVB in eyes with VH/PDR, eyes scheduled for PPV within one month of the index date were 
also excluded, as their natural history was likely predetermined at the time of the initial injection. Finally, subjects with less than one year of follow-up were excluded as well.

\section{Data Abstraction}

Study subject's medical records were reviewed for demographic information and dates and types of clinically relevant diagnoses, injection(s), PRP, surgery and resolution of VH status. Data were recorded for at least one year of follow-up, and up to two years if PPV was not performed within the first year. VA on presentation and at one year was also recorded for all eligible eyes. VA measurements were converted to logarithm of the minimum angle of resolution (LogMAR) for analysis. As previously described ${ }^{12}$, hand motion VAs were assigned a LogMAR value of 3.00 (20/20000), while any VAs recorded as light perception were excluded from visual acuity analysis.

\section{Outcomes}

The primary outcome of interest in this study was the rate of PPV among subjects after IVB injection. We also recorded any additional treatments, IVB injections and PRP performed within a one-year follow-up period. Criteria for additional treatment (after initial injection) was determined at the discretion of the treating physician with consideration for the patient's desires for their care. Subjects were further subdivided into those with PRP prior to the study and treatment naïve (no history of PRP) and additionally evaluated. Secondary analyses included change in VA over study duration. Finally, for those patients who did not have a PPV and had two years of follow-up, data were collected to determine what proportion of eyes required additional treatment and the types of management that occurred in the second year.

\section{Statistical Analysis}

Demographic characteristics taken at the time of initial presentation were analyzed using means and standard deviations for continuous variables and frequencies and percentages for categorical variables. Student t-tests were used for comparisons of continuous variables and chi-squared tests were used for categorical variables. All statistical analyses were performed with STATA14® (College Station, TX). Two-sided p-values $<0.05$ were considered statistically significant.

\section{RESULTS}

Of the 474 patients reviewed who had a diagnosis of diabetes and treatment with IVB, 111 eyes of 95 patients were eligible for the study following application of inclusion and exclusion criteria. Reasons for exclusion included prior treatment with anti-VEGF therapy for any indication prior to the study start date $(n=183)$, less than one year of follow-up $(n=111)$, concurrent and future diagnosis of DME $(n=25)$, prior PPV $(n=18)$, NVG $(n=14)$, and PPV performed within one month of initial injection $(n=12)$. Baseline characteristics of the eligible study population are listed in Table 1.

Figure 1 is the flow chart representing the interventions performed on the 111 eligible eyes over the first year following presentation with $\mathrm{VH}$. The overall average number of injections 
was 2.0 (range: 1 to 9) injections per eye. Of the 111 eyes, a total of 21 (18.9\%) underwent PPV within one year. Of these 21 eyes, $12(10.8 \%)$ had additional treatments including injections $(n=7), \operatorname{PRP}(n=2)$, or both $(n=3)$ during the first year. The average number of injections in the 21 vitrectomized eyes was 1.6 (range: 1 to 3 ) per eye. The reasons for vitrectomy in this group were recurrent/non-clearing vitreous hemorrhage (NCVH) in 17 eyes, NCVH/TRD in 2 eyes and TRD in 2 eyes.

A total of 35 (31.6\%) eyes were treated with injections alone over the course of the first year and the average number of injections in this group was 2.4 (range: 1 to 9 ) per eye. Of note, $13(11.7 \%)$ eyes did not receive any additional intervention other than the initial IVB during the first year.

Lastly, 55 (49.5\%) eyes received PRP treatment at some point during the first year. Many of these patients also received additional injections $(n=12)$, supplemental PRP treatments $(\mathrm{n}=10)$, or both $(\mathrm{n}=14)$. The average number of injections in this group was 1.8 (range: 1 to 5) per eye.

Figure 2 demonstrates the natural history of treatment naïve eyes $(n=43,38.7 \%)$ through one year of follow up. PPV was performed on eight (18.6\%) of the treatment naïve eyes.

Multiple IVB injections were required in four $(9.3 \%)$ of these eyes, while the remaining four eyes received no additional treatment other than the initial IVB injection prior to the PPV procedure. The average number of injections in the eight vitrectomized eyes was 1.5 (range: 1 to 2) per eye.

Of the 43 treatment naïve eyes, seven (16.3\%) were treated with injections alone over the course of the first year and the average number of injections in this group was 2.0 (range: 1 to 4) per eye. No additional intervention other than the initial IVB was required for three (7.0\%) eyes during the first year.

PRP treatment was administered to $28(65.1 \%)$ of the 43 eyes at some point during the first year. A portion of these patients also received supplemental PRP $(n=8)$, additional injections $(n=6)$, or both $(n=6)$. The average number of injections in this group was 1.7 (range: 1 to 5) per eye.

In the 68 eyes that had prior PRP (Figure 3), PPV was performed in a total of $13(19.1 \%)$. Additional treatments, such as injections $(n=3), \operatorname{PRP}(n=2)$, or both $(n=3)$ were performed in eight $(11.8 \%)$ eyes during the first year. The average number of injections in the 13 vitrectomized eyes in this subset was 1.7 (range: 1 to 3 ) per eye.

A total of 28 (41.2\%) eyes were treated with injections alone over the course of the first year and the average number of injections in this group was 2.5 (range: 1 to 9) per eye. Of note, $10(14.7 \%)$ eyes with prior PRP did not receive any additional intervention other than the initial IVB during the first year.

Lastly, 27 (39.7\%) eyes received additional PRP treatment at some point during the first year. Supplementary injections $(n=6)$, PRP treatments $(n=2)$, or both $(n=8)$ were performed 
in 16 of these eyes. The average number of injections in this group was 1.9 (range: 1 to 5) per eye.

A second year of data was available on $69(76.7 \%)$ of the 90 eyes that did not have a vitrectomy within the first year of follow-up. Figure 4 represents all interventions administered to each of these 69 eyes through two years of follow-up. At two years, 10 (14.5\%) had undergone a PPV. All 10 eyes had additional treatments prior to PPV including injections ( $n=5)$, PRP $(n=1)$, and both $(n=4)$. Vitrectomized eyes required an average of 3.7 (range: 1 to 5) injections per eye through two years.

Injections alone were used to manage $16(23.2 \%)$ of the 69 eyes through two years with an average of 3.1 (range: 1 to 12 ) injections administered per eye. Of note, six (8.7\%) eyes did not receive any additional intervention within two years other than the initial IVB injection.

PRP treatment was performed in 43 (62.3\%) eyes at some point during the two years of follow-up. Many of these patients also received additional injections ( $n=9)$, supplemental PRP treatments $(n=9)$, or both $(n=17)$. The average number of injections in this group was 2.7 (range: 1 to 9) per eye.

Among the 111 eyes in the study, recurrent VH was documented in 19 (17.1\%) eyes during the first year of follow-up. PRP was performed in 12 (10.8\% of 111 eyes) of these eyes, six $(5.4 \%)$ of which required multiple sessions. Multiple injections were required in eleven (9.9\%) of the 12 eyes as well. The remaining seven (6.3\%) eyes with recurrent VH did not require additional treatment other than additional IVB injections. During the second year of follow up, four (3.6\%) of eyes with recurrent VH required PPV.

Survival analysis was performed while accounting for censoring due to competing outcomes for any second treatment (injection, PRP or PPV) and compared to needing a second injection only. The Kaplan Meier curve for the survival estimates is seen in Figure 5. An additional survival analysis was performed again censoring for competing outcomes this time comparing treatment naïve eyes to those with prior PRP (Figure 6). No statistical difference was seen between the curves $(\mathrm{p}=0.98)$.

Visual acuities were recorded for each eye on presentation and at one year of follow-up. Table 2 compares VA outcomes (logMar [Snellen equivalent]) under various criteria. Two eyes were excluded from analysis due to "light perception" visual acuity. Eyes that underwent PPV were found to have worse vision at baseline (1.4 [20/502] vs 0.91 [20/163]; $P=0.031)$ and after one year of follow-up $(0.89$ [20/155] vs 0.45 [20/56]; $P=0.003)$ than those that did not have a vitrectomy. There were strong trends for better VA at baseline in eyes who received PRP during the study period ( 0.85 [20/142] vs 1.2 [20/317]; $P=0.051$ ), as well as after one year of follow-up $(0.44$ [20/55] vs 0.64 [20/87; $P=0.076)$. There was no significant difference in VA between eyes that received any intervention (PRP or PPV) and those that were managed with injections only before $(P=0.610)$ or after $(P=0.465)$ the oneyear follow-up. Similarly, eyes treated with PRP prior to the initial IVB injection and those that were treatment naïve had no significant difference in VA before $(P=0.950)$ or after $(P=0.918)$ the study. The overall change/improvement in VA from baseline to one year was not found to be significantly different in any group ( $\mathrm{P}>0.05$ for all comparisons). 


\section{DISCUSSION}

The purpose of this study was to evaluate the natural history and possible treatment courses for patients who received IVB for VH due to PDR. We did not aim to prove one treatment was better than another. Nor was it our objective to determine how many or how frequent injections should be performed in order to obtain a satisfactory clearing of VH which previously has been the end point of many PDR and VH studies. In our observational dataset, physician and patient preferences (including aversion to various treatment options) often dictate treatment course in a real world clinical setting, not a pre-determined clinical trial regimen (i.e. the timing of a PPV in a monocular patient can be very different from one for a binocular patient). This treatment variability is what allowed us to better elucidate the natural history of these patients.

In our study, we found that of the 111 eyes, $72.1 \%$ were managed with anti-VEGF and PRP alone through two years. The PPV rate (27.9\%) was higher than that reported in the DRCR.net Protocol S (4\% ranibizumab group vs. $15 \%$ PRP group) ${ }^{10}$. However, in contrast to our study, VH was not a requirement for inclusion in the DRCR study and was only present in a portion of patients (27\% ranibizumab group vs. $34 \%$ PRP group $)^{10}$. Conversely, the only other popularly cited report on VH clearance rate is from the Diabetic Retinopathy Vitrectomy Study (DRVS), which was published in 1985 and found 80\% of patients did not clear spontaneously and required surgery. Comparison of this study to ours however, is difficult due to improvements in systemic DM control, ubiquity of laser treatments and their mandated requirement for $\mathrm{VH}$ to cause $5 / 200$ vision or less ${ }^{16}$.

Also of interest, only 4 (3.6\%) eyes of the 111 required surgery for a TRD. Previous reports have expressed concern for "crunch" with anti-VEGF use for PDR and typically have shown a higher rate of TRD formation (5.2\% of 211 eyes) than seen in this study ${ }^{14,15}$. Our data, however, suggests that TRD occurrence can be lower in a real-world population, albeit with the caveat of proper patient selection. Additionally, in contrast to previous reports, our study excluded eyes with pre-existing TRDs, which may account for this discrepancy. Future studies will be needed to determine which patients are most or least likely to be at high risk for TRD formation.

We found that 35 (31.6\%) eyes in our study were treated with injections alone over the course of the first year. Those injections were not given monthly, but on pro re nata basis as judged by the provider. Although as many as nine injections were given in the first year, the average was only 2.4 , and 13 of 35 eyes (37.1\%) received only the initial injection. It should be pointed out, however, that 10 of the 13 (76.9\%) eyes that required only one injection had PRP prior to the study. These results indicate that a proportion of patients with VH/PDR (often those with prior PRP) may only require minimal intervention with one or two antiVEGF injections. Based on these findings, requiring a patient to undergo a fixed regimen of injections may subject the patient to unnecessary costs and treatment burden. Additional studies focusing on improved identification of specific patients that are likely to be require less intensive care may be of clinical interest. 
The overall findings in the treatment naïve group vs. the prior PRP group were consistent with expectations. Not surprisingly, the proportion of subjects undergoing PRP (65.1\% vs. $39.7 \%$ ) was higher in the treatment naïve group, while the number of injections only (16.3\% vs. 41.2\%) was higher in the prior PRP group. Interestingly, however, the rate of PPV was very similar (18.6\% treatment naïve vs. $19.1 \%$ prior PRP) between the two groups.

Several limitations should be considered during interpretation of these results. This study was designed as a natural history study and therefore did not have a control group to make comparative treatment statements. Additionally, physician and patient preference rather than a set regimen or protocol determined the course of management, which may have introduced bias. Eligible eyes were examined by at least one of the four retinal specialists at the Scheie Eye Institute, all of which practice under varying modus operandi. Finally, the study represents findings from a single institution with a limited number of physicians and may not be generalizable to other populations.

In conclusion, our results corroborate that there is a role for anti-VEGF injections in patients with VH in the setting of PDR. Given the results and stated limitations of the study, a randomized controlled trial would help clarify this role, determine the most appropriate dosing regimen, and identify patients that may require minimal intervention to help alleviate treatment burden.

\section{Supplementary Material}

Refer to Web version on PubMed Central for supplementary material.

\section{Acknowledgments}

Funding/Support: National Institutes of Health K23 Award (BVB) (1K23EY025729 - 01); Additional funding was provided by Research to Prevent Blindness and the Paul and Evanina Mackall Foundation. Funding from each of the above sources was received in the form of block research grants to the Scheie Eye Institute. None of the organizations had any role in the design or conduction of the study.

\section{References}

1. Sinawat S, Rattanapakorn T, Sanguansak T, Yospaiboon Y. Intravitreal bevacizumab for proliferative diabetic retinopathy with new dense vitreous hemorrhage after full panretinal photocoagulation. Eye (Lond). 2013; 27(12):1391-1396. [PubMed: 24037235]

2. Michels RG. Proliferative diabetic retinopathy: pathophysiology of extraretinal complications and principles of vitreous surgery. Retina. 1981; 1(1):1-17. [PubMed: 15633404]

3. Yang CS, Hung KC, Huang YM, Hsu WM. Intravitreal bevacizumab (Avastin) and panretinal photocoagulation in the treatment of high-risk proliferative diabetic retinopathy. J Ocul Pharmacol Ther. 2013; 29(6):550-555. [PubMed: 23495932]

4. Gupta OP, Weichel ED, Regillo CD, et al. Postoperative complications associated with 25-gauge pars plana vitrectomy. Ophthalmic Surg Lasers Imaging. 2007; 38(4):270-275. [PubMed: 17674916]

5. Steel DH, Connor A, Habib MS, Owen R. Entry site treatment to prevent late recurrent postoperative vitreous cavity haemorrhage after vitrectomy for proliferative diabetic retinopathy. $\mathrm{Br}$ J Ophthalmol. 2010; 94(9):1219-1225. [PubMed: 19955203]

6. Simunovic MP, Maberley DA. Anti-vascular endothelial growth factor therapy for proliferative diabetic retinopathy: a systematic review and meta-analysis. Retina. 2015; 35(10):1931-1942. [PubMed: 26398553] 
7. Bhavsar AR, Torres K, Beck RW, et al. Randomized clinical trial evaluating intravitreal ranibizumab or saline for vitreous hemorrhage from proliferative diabetic retinopathy. JAMA Ophthalmol. 2013; 131(3):283-293. [PubMed: 23370902]

8. Bhavsar AR, Torres K, Glassman RA, et al. Evaluation of results 1 year following short-term use of ranibizumab for vitreous hemorrhage due to proliferative diabetic retinopathy. JAMA Ophthalmol. 2014; 132(7):889-890. [PubMed: 25010170]

9. Landa G, Amde W, Doshi V, et al. Comparative study of intravitreal bevacizumab (Avastin) versus ranibizumab (Lucentis) in the treatment of neovascular age-related macular degeneration. Ophthalmologica. 2009; 223(6):370-375. [PubMed: 19590252]

10. Gross JG, Glassman AR, Jampol LM, et al. Panretinal photocoagulation vs intravitreous ranibizumab for proliferative diabetic retinopathy: A randomized clinical trial. JAMA. 2015; 314(20):2137-2146. [PubMed: 26565927]

11. Spaide RF, Fisher YL. Intravitreal bevacizumab (Avastin) treatment of proliferative diabetic retinopathy complicated by vitreous hemorrhage. Retina. 2006; 26(3):275-278. [PubMed: 16508426]

12. Holladay JT. Proper method for calculating average visual acuity. J Refract Surg. 1997; 13:388391. [PubMed: 9268940]

13. Arevalo JF, Lasave AF, Wu L, et al. Intravitreal bevacizumab for proliferative diabetic retinopathy: results from the pan-american collaborative retina study group (PACORES) at 24 months of follow-up. Retina.

14. Arevalo JF, Maia M, Flynn HW Jr, et al. Tractional retinal detachment following intravitreal bevacizumab (Avastin) in patients with severe proliferative diabetic retinopathy. Br J Ophthalmol. 2008; 92(2):213-216. [PubMed: 17965108]

15. Nakao S, Ishikawa K, Yoshida S, et al. Altered vascular microenvironment by bevacizumab in diabetic fibrovascular membrane. Retina. 2013; 33(5):957-963. [PubMed: 23503340]

16. Diabetic Retinopathy Vitrectomy Study Research Group. Early vitrectomy for severe vitreous hemorrhage in diabetic retinopathy. Two-year results of randomized trial, Diabetic retinopathy vitrectomy study report 2. Arch Ophthalmol. 1985; 103:1644-1652. [PubMed: 2865943] 


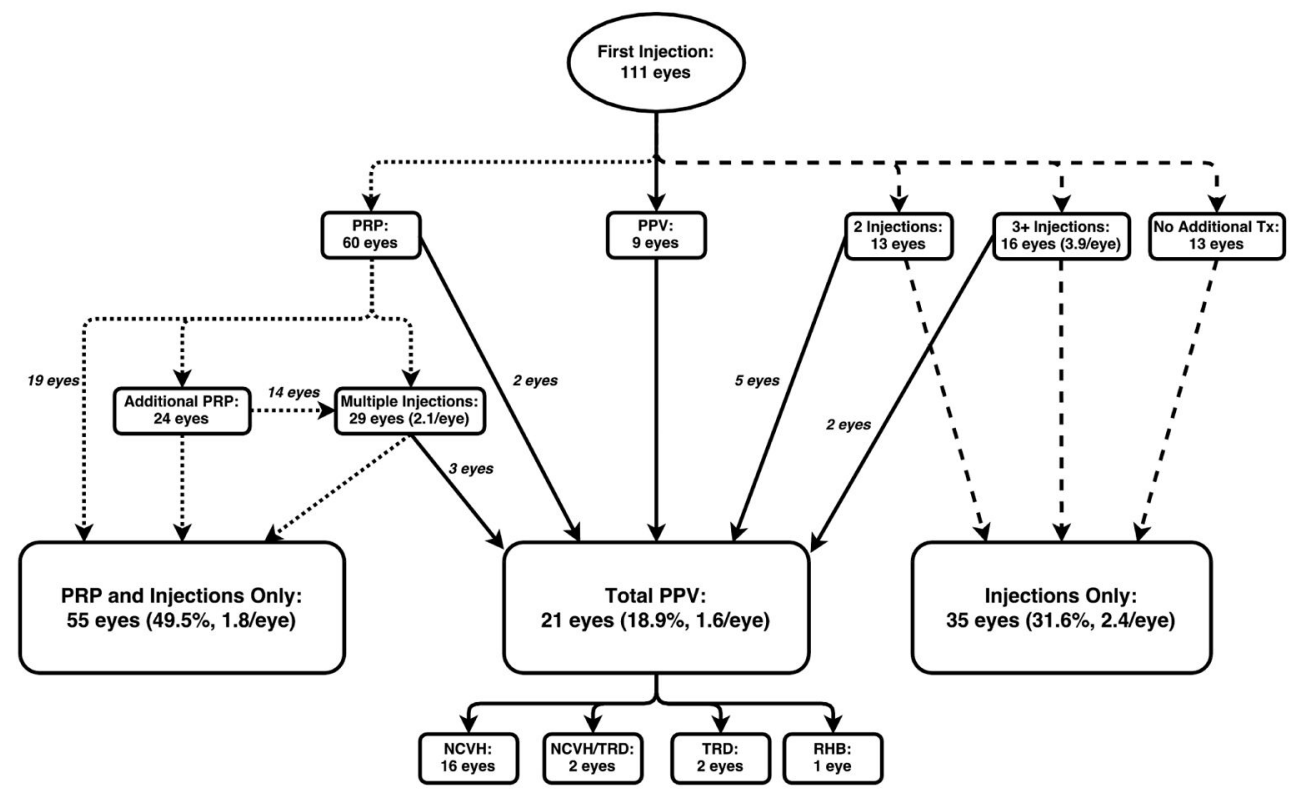

Key: PPV: pars plana vitrectomy; PRP: panretinal photocoagulation; NCVH: non-clearing vitreous hemorrhage; RHB: recurrent bleeding; TRD: tractional retinal detachment; Tx: treatment; (\#/eye): average number of injections per eye in subset

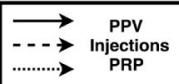

FIGURE 1.

Flowchart of patients after intravitreal bevacizumab injection for vitreous hemorrhage (VH) in the setting of proliferative diabetic retinopathy (PDR) at one year. 


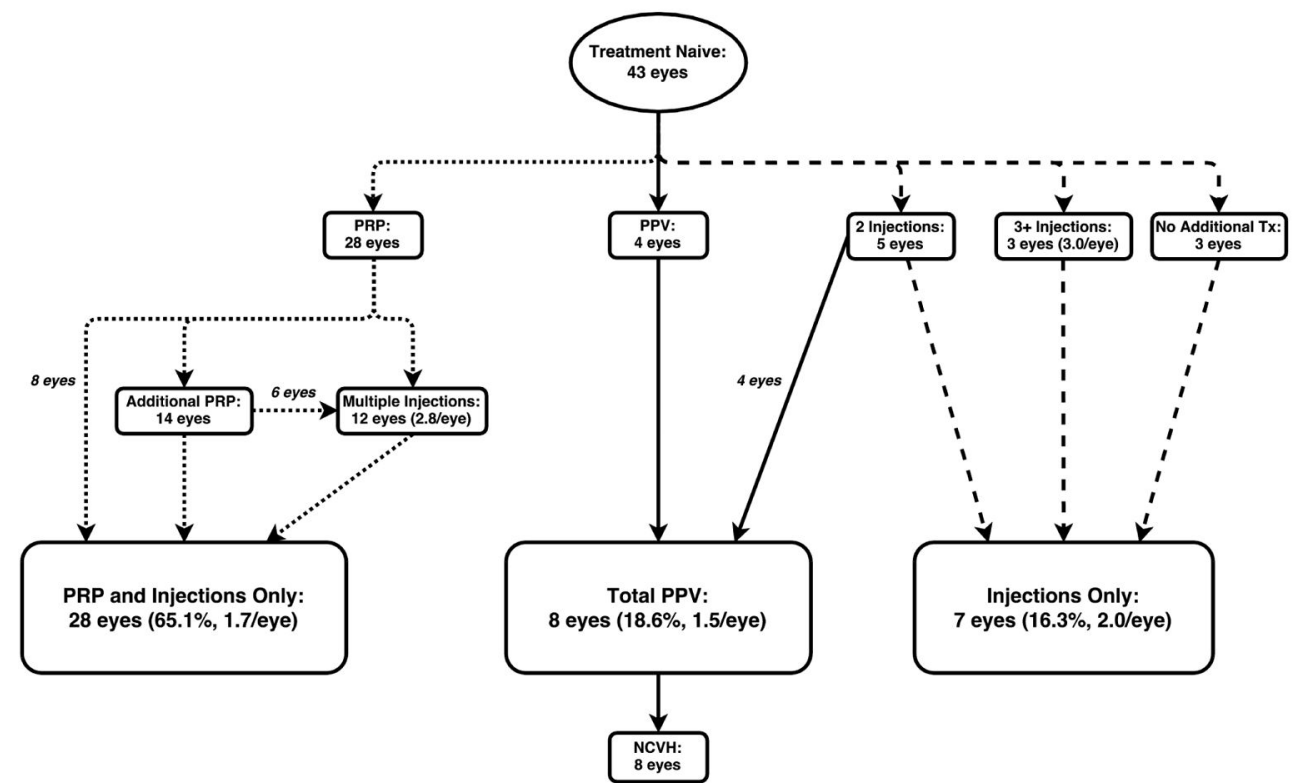

Key: PPV: pars plana vitrectomy; PRP: panretinal photocoagulation; NCVH: non-clearing vitreous hemorrhage; RHB: recurrent bleeding; TRD: tractional retinal detachment; Tx: treatment; (\#/eye): average number of injections per eye in subset

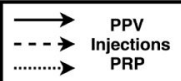

FIGURE 2.

Flowchart of treatment naïve patients after intravitreal bevacizumab injection for vitreous hemorrhage (VH) in the setting of proliferative diabetic retinopathy (PDR). 


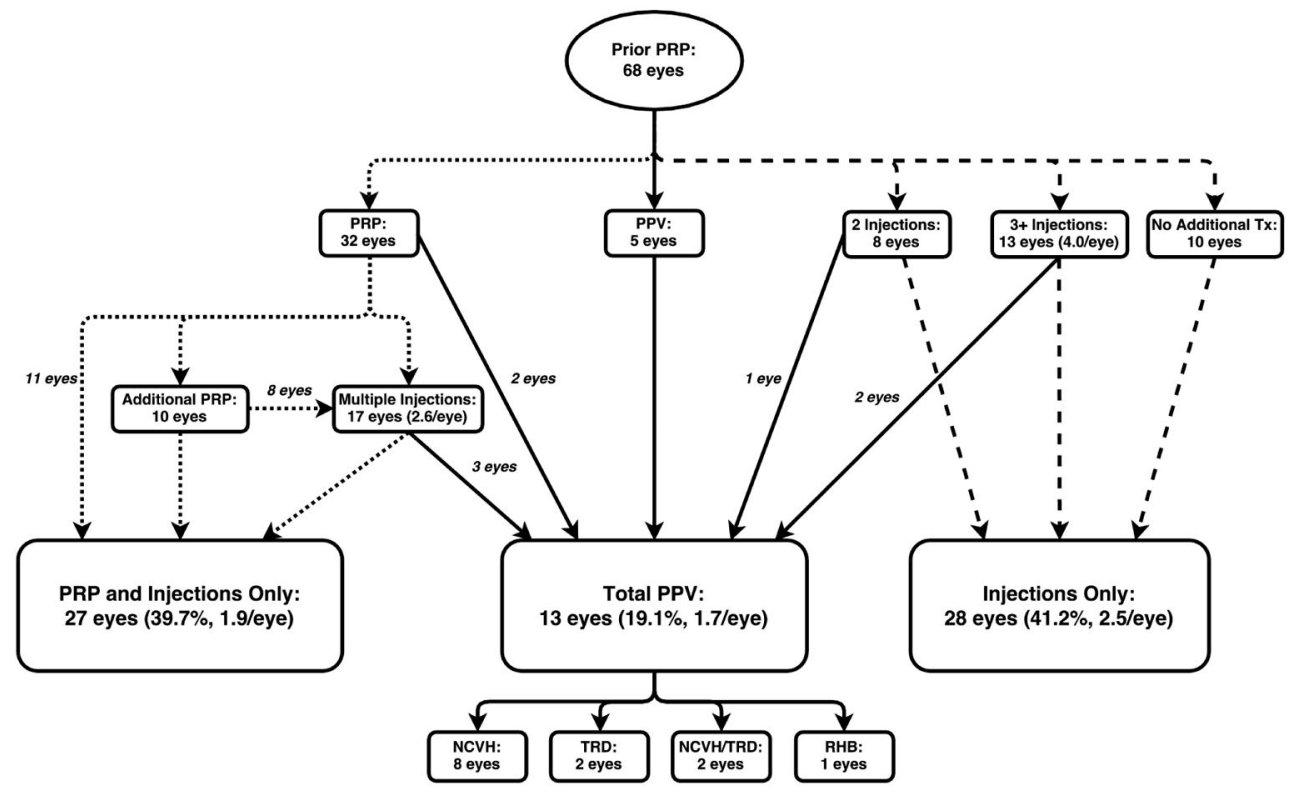

Key: PPV: pars plana vitrectomy; PRP: panretinal photocoagulation; NCVH: non-clearing vitreous hemorrhage; RHB: recurrent bleeding; TRD: tractional retinal detachment; Tx: treatment; (\#/eye): average number of injections per eye in subset

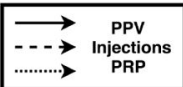

FIGURE 3.

Flowchart of patients with prior panretinal photocoagulation (PRP) after intravitreal bevacizumab injection for vitreous hemorrhage $(\mathrm{VH})$ in the setting of proliferative diabetic retinopathy (PDR). 


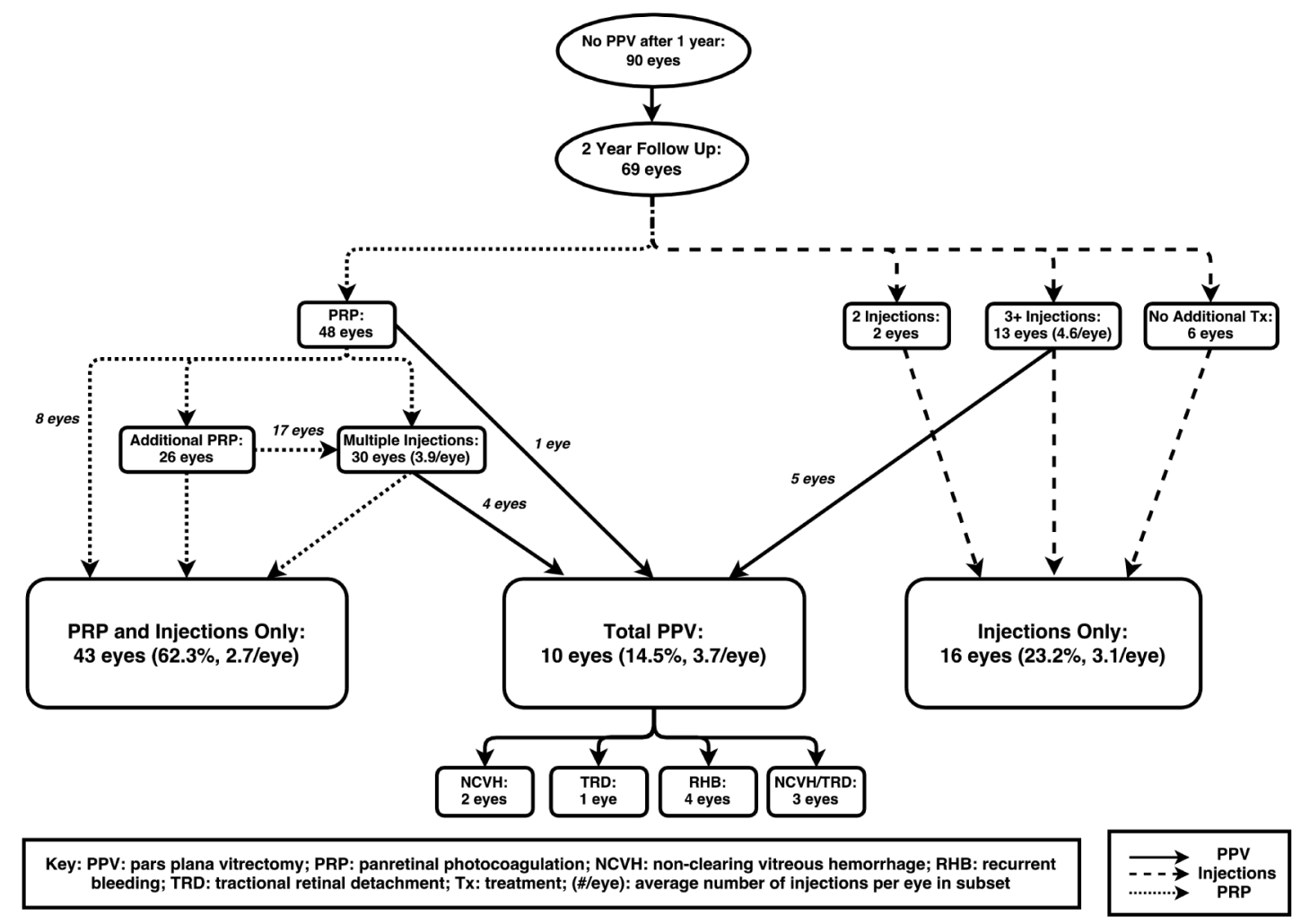

FIGURE 4.

Flowchart at two years follow-up of patients after intravitreal bevacizumab injection for vitreous hemorrhage ( $\mathrm{VH})$ in the setting of proliferative diabetic retinopathy (PDR) who did not undergo pars plana vitrectomy (PPV) in year one. 


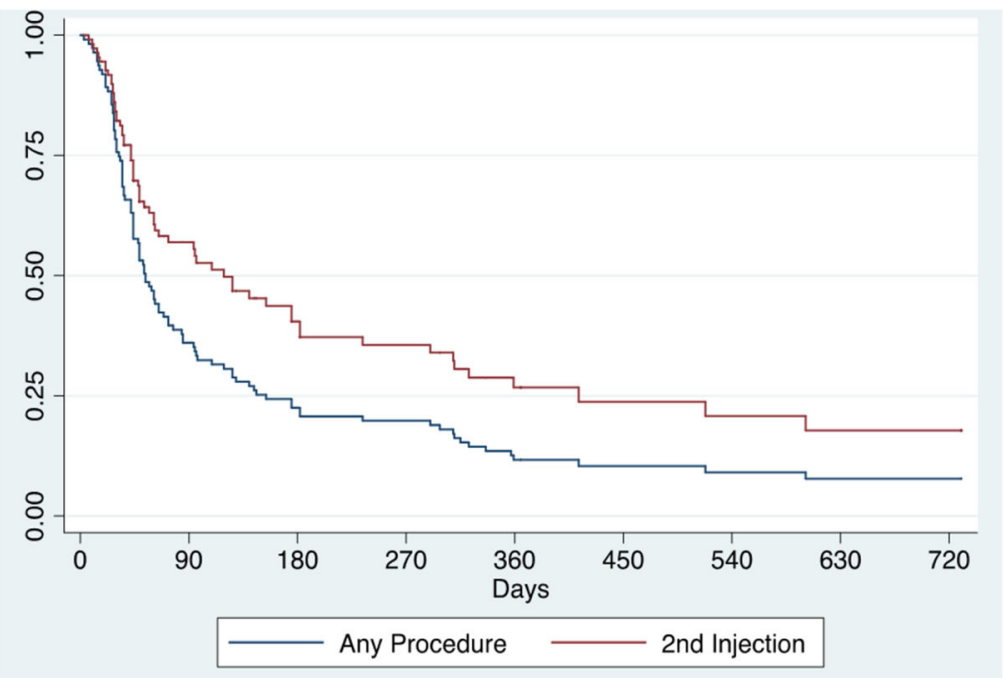

FIGURE 5.

Kaplan Meier curve for survival estimates for second injection only versus any additional treatment (injection, panretinal photocoagulation (PRP), pars plana vitrectomy (PPV)) in patients with vitreous hemorrhage $(\mathrm{VH})$ in the setting of proliferative diabetic retinopathy (PDR). 


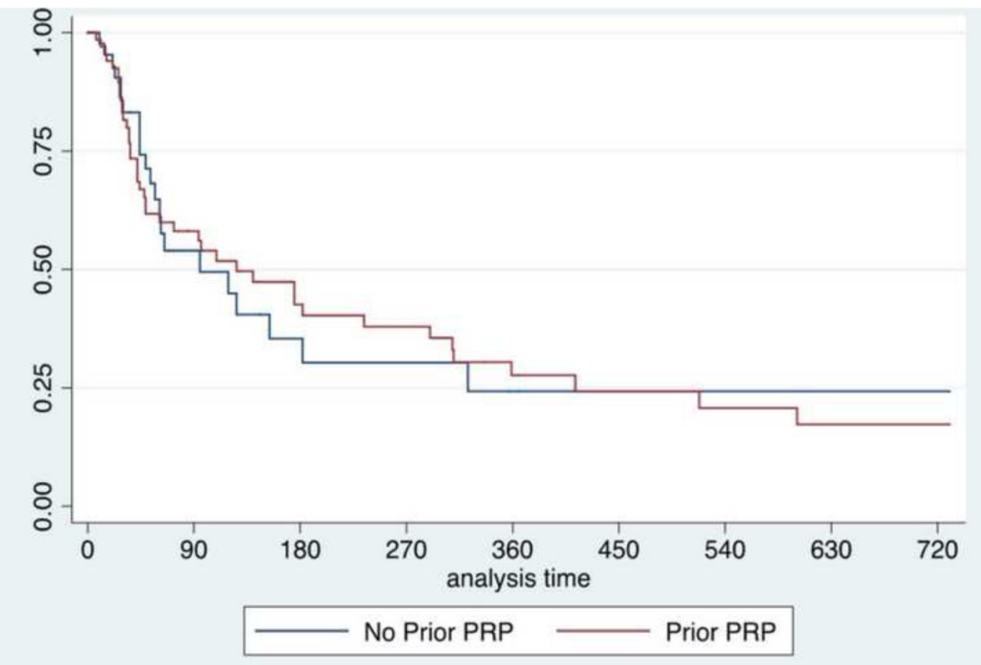

FIGURE 6.

Kaplan Meier curve for survival estimates for treatment naïve eyes (no prior panretinal photocoagulation (PRP)) versus eyes with prior PRP in patients with vitreous hemorrhage $(\mathrm{VH})$ in the setting of proliferative diabetic retinopathy (PDR). 


\section{Table 1}

Baseline characteristics of patients after intravitreal bevacizumab injection for vitreous hemorrhage in the setting of proliferative diabetic retinopathy

\begin{tabular}{|c|c|}
\hline Characteristics & No. Eyes \\
\hline Eligible for study & 111 \\
\hline Age, median $\left(\mathrm{SD}^{a}\right)$ & $63(11.99)$ \\
\hline \multicolumn{2}{|l|}{ GENDER } \\
\hline Male (\%) & $53(47.7)$ \\
\hline Female (\%) & $58(52.3)$ \\
\hline \multicolumn{2}{|l|}{ RACE } \\
\hline Black (\%) & $74(66.7)$ \\
\hline White (\%) & $29(26.1)$ \\
\hline Asian (\%) & $3(2.7)$ \\
\hline Hispanic Latino (\%) & $3(2.7)$ \\
\hline Undisclosed (\%) & $2(1.8)$ \\
\hline
\end{tabular}

$a_{\text {standard deviation }}$ 
Table 2

Visual acuity analysis of patients after intravitreal bevacizumab injection for vitreous hemorrhage in the setting of proliferative diabetic retinopathy

\begin{tabular}{|c|c|c|c|}
\hline & PPV $b$ & No PPV & $P$-value \\
\hline No. eyes & 20 & 89 & \\
\hline VA VA $^{a}$ Baseline & & & 0.031 \\
\hline Snellen equivalent, mean & $20 / 502$ & $20 / 163$ & \\
\hline $\log M A R$, mean $\left(S D^{d}\right)$ & $1.4(1.0)$ & $0.91(0.76)$ & \\
\hline $95 \%$ confidence interval & 0.87 to 1.8 & 0.75 to 1.1 & \\
\hline VA@ 1 Year & & & 0.003 \\
\hline Snellen equivalent, mean & $20 / 155$ & $20 / 56$ & \\
\hline $\log M A R$, mean $(S D)$ & $0.89(0.78)$ & $0.45(0.53)$ & \\
\hline \multirow[t]{2}{*}{$95 \%$ confidence interval } & 0.53 to 1.3 & 0.34 to 0.56 & \\
\hline & $\mathbf{P R P}^{c}$ & No PRP & $P$-value \\
\hline No. eyes & 59 & 50 & \\
\hline VA @ Baseline & & & 0.051 \\
\hline Snellen equivalent, mean & $20 / 142$ & $20 / 317$ & \\
\hline $\log M A R$, mean $(S D)$ & $0.85(0.82)$ & $1.2(0.83)$ & \\
\hline $95 \%$ confidence interval & 0.64 to 1.1 & 0.93 to 1.4 & \\
\hline VA@ 1 Year & & & 0.076 \\
\hline Snellen equivalent, mean & $20 / 55$ & $20 / 87$ & \\
\hline $\log M A R$, mean $(S D)$ & $0.44(0.56)$ & $0.64(0.63)$ & \\
\hline \multirow[t]{2}{*}{ 95\% confidence interval } & 0.29 to 0.58 & 0.46 to 0.82 & \\
\hline & Any Intervention & Injection Only & $P$-value \\
\hline No. eyes & 74 & 35 & \\
\hline VA @ Baseline & & & 0.610 \\
\hline Snellen equivalent, mean & $20 / 187$ & $20 / 252$ & \\
\hline $\log M A R$, mean $(S D)$ & $0.97(0.89)$ & $1.1(0.71)$ & \\
\hline $95 \%$ confidence interval & 0.76 to 1.2 & 0.81 to 1.3 & \\
\hline VA @ 1 Year & & & 0.465 \\
\hline Snellen equivalent, mean & $20 / 73$ & $20 / 59$ & \\
\hline $\log M A R$, mean $(S D)$ & $0.56(0.67)$ & $0.47(0.43)$ & \\
\hline \multirow[t]{2}{*}{$95 \%$ confidence interval } & 0.41 to 0.72 & 0.32 to 0.62 & \\
\hline & Prior PRP & Treatment Naïve & $P$-value \\
\hline No. eyes & 66 & 43 & \\
\hline VA @ Baseline & & & 0.950 \\
\hline Snellen equivalent, mean & $20 / 195$ & $20 / 200$ & \\
\hline $\log M A R$, mean $(S D)$ & $0.99(0.82)$ & $1.0(0.86)$ & \\
\hline 95\% confidence interval & 0.79 to 1.2 & 0.74 to 1.3 & \\
\hline
\end{tabular}

Am J Ophthalmol. Author manuscript; available in PMC 2018 April 01. 


\begin{tabular}{|c|c|c|c|}
\hline & PPV $^{\boldsymbol{b}}$ & No PPV & P-value \\
\hline VA @ 1 Year & & & 0.918 \\
\hline Snellen equivalent, mean & $20 / 68$ & $20 / 69$ & \\
\hline LogMAR, mean (SD) & $0.53(0.58)$ & $0.54(0.64)$ & \\
\hline 95\% confidence interval & 0.38 to 0.67 & 0.38 to 0.67 & \\
\hline
\end{tabular}

"VA: visual acuity

$b_{\text {PPV: pars plana vitrectomy }}$

$c_{\text {PRP: panretinal photocoagulation }}$

${ }^{d}$ SD: standard deviation

Note - Two eyes were excluded from analysis due to "light perception" visual acuity 